\title{
Optimized Voting Scheme for Efficient Vanishing Point Detection in General Road Images
}

\author{
Vipul H.Mistry \\ Ph.D. student, Electronics \& Communication Engineering \\ Department, \\ Uka Tarsadia University, Surat, India
}

\author{
Ramji Makwana \\ Professor \& Head, Department of Information Technology, \\ V.V.P. Engineering College, \\ Rajkot, India
}

\begin{abstract}
Next generation automobile industries are aiming for development of vision-based driver assistance system and driver-less vehicle system. In the context of this application, a major challenge lies in the identification of efficient road region segmentation from captured image frames. Recent research work suggests that use of a global feature like vanishing point makes the road detection algorithm more robust and general for all types of roads. The goal of this research work is the reduction of computational complexity involved with voting process for identification of vanishing point. This paper presents an efficient and optimized voter selection strategy to identify vanishing point in general road images. The major outcome of this algorithm is the reduction in computational complexity as well as improvement in efficiency of vanishing point detection algorithm for all types of road images. The key attributes of the methodology are dominant orientation selection, voter selection based on voter location and modified voting scheme, combining dominant orientation and distance based soft voting process. Results of a number of qualitative and quantitative experiments clearly demonstrate the efficiency of proposed algorithm.
\end{abstract}

Keywords-Road Detection; Vanishing Point; Gabor Filter; voting scheme; general road segmentation

\section{INTRODUCTION}

Vision-based autonomous navigation is the future of automobile industry. Some companies have already incorporated features like driver assistance system based on GPS and various sensors. The future of this advancement is vision based driverless vehicle car. One of the major constraint in the development vision based driverless car is the efficient detection of road area. With advancements in proximity sensors, it is possible to detect roads but still vision based approach is one which can enhance the usefulness of the system to a great extent. With proximity sensors, one cannot understand nearby environment and recognize various properties of the surface on which vehicle is moving. The only solution is a vision based approach where a car can recognize the road surface with the help of the camera mounted on it. This camera will work like the human eye and provide the scene information regarding images. The task is now reduced to understand this 2-D image and extract information required as per various applications. In last decade researchers have done considerable work in this area and suggested some good approaches for identification of road surface from a given image. These approaches mainly focus on extraction of features that can help to distinguish between the road and offroad regions.
All road segmentation algorithms are mainly classified into two categories: region properties based approaches [1-7] and boundary based approaches [8-11]. Various road types i.e. structured, unstructured, rural roads and highways along with various surrounding environments and day light conditions make the task more challenging for both previously suggested methods. The solution is a use of vanishing point. The vanishing point of an image provides a strong cue for road identification and makes the algorithm general for all types of roads with various surrounding environments. The goal of this research paper is to suggest optimized voter selection strategy based voting process that will reduce the computational complexity of existing vanishing point detection algorithm and enhance the accuracy of the same. Rest of the paper is organized as follows: Section II highlights major contributions by various researchers in vanishing point detection and draws concluding remarks to motivate further scope of research work. Section III describes proposed optimum voter selection strategy. Section IV describes modified voting process for vanishing point detection. Section $\mathrm{V}$ demonstrates the efficiency and effectiveness of the algorithm based on various qualitative and quantitative experiments.

\section{LITERATURE SURVEY}

To overcome the challenges introduced by semi-structured and unstructured roads, the concept of vanishing point detection came into existence. The majority of vanishing point detection methods rely on line segments in the image. The task of detecting those vanishing points, which correspond to the dominant directions of a scene, is traditionally solved in two steps. Firstly, line segments are clustered together and then vanishing point is selected under the condition that a cluster of line segments share a common vanishing point. A different approach for reducing the computational complexity of the accumulation step is to apply the Hough transformation, by mapping the parameters of the line segments into a bounded Hough space [9, 11]. Tuytelaars et al. [11] applied the Hough transformation three times (Cascade Hough transformation). At different levels of the Cascade Hough transformation a peak in the Hough space corresponds to a vanishing point and a vanishing line respectively.

Rasmussen et. al. [14-15] replaced edge detection step with dominant orientation identification using texture properties. This method greatly influenced the research work towards identification of vanishing point estimation. Most of the existing vanishing point detection methods rely on three 
steps: extraction of dominant orientations, identification of any line segments in the image and voting procedure for vanishing point estimation [14-17]. To achieve precise orientation estimation, one needs to apply a large number of orientation filters in all possible directions from $0^{\circ}$ to $180^{\circ}$. Designing and applying a bank of differently rotated filters is computationally expensive. To address this problem Freeman and Adelson [13] proposed a steerable filter in which each arbitrarily oriented filter can be formed by a linear combination of a fixed set of basis-oriented filters. But it still requires steering the basis-oriented filters in all directions with a precision of $1^{\circ}$. In 2010, Hui Kong et. al. [17] came up with a general road detection method from a single image using Vanishing point detection approach. He suggested 36 orientations Gabor Filters for extraction of orientation information at each edge pixel. Distance based soft voting procedure suggested in this paper also made the detection process more robust and increased the efficiency in vanishing point detection.

In 2012, Peyman Moghadam et. al. [18] proposed a novel methodology based on image texture analysis for the fast estimation of the vanishing point detection in the challenging environments. The key attributes of the methodology consist of the optimal local dominant orientation method that uses joint activity of four Gabor filters followed by an efficient and robust voting scheme for real-time detection of the vanishing point. Estimation of vanishing point greatly enhances the robustness and effectiveness of road detection for all type of roads, irrespective to structured roads with lane markings, semi- structured roads without lane markings and unstructured roads with improper road regions. Combining the efficiency of edge-based methods and the orientation coherence concept of texture-based methods Xin Lu [20] proposed a more efficient method for vanishing point detection in 2012. In 2014, Cheng $\mathrm{Xu}$ et. al. [21] introduced a concept of dominant road orientation based voting in place of soft voting methods used by Hui Kong et al. [16-17] and Peyman Moghadam et al [18].

The accuracy of vanishing point detection methods discussed so far mainly depends on three parameters: efficiency of orientation extraction, effective voter selection and candidate selection for vanishing point to be used during voting process. The motivation of this research work is to suggest an optimum method for voter selection and use of only most effective voters during the voting process. This will enhance the speed of the algorithm, and it will also increase the efficiency of the existing algorithm. Major contributions of this research paper are listed as follows:

- Optimum voter selection based on dominant orientations of an image.

- Removal of outliers based on edge map and horizon line detection.

- Strategy for voter selection and candidate vanishing point selection.

- Modified locally adaptive soft voting scheme with dominant orientation information.
Next section describes the fundamental steps for vanishing point detection and also highlights the ways to make the algorithm more efficient and fast.

\section{Proposed Optimum VANishing Point AND Voter CANDIDATE SELECTION}

The vanishing point plays an important role as a global constraint for detecting road direction, since all parallel border road lines and road edges converge into a single vanishing point [14]. The basic steps for vanishing point detection are orientation extraction, selection of candidate pixels as vanishing point candidates and voting process.

\section{A. Selection of Dominant Orientations}

In this paper, 36 Gabor orientation Filters are used to extract orientation information at each pixel. A Gabor filter can be viewed as a sinusoidal plane of particular frequency and orientation, modulated by a Gaussian envelope. The kernels of the Gabor filters are similar to the 2-D receptive field profiles of the mammalian cortical simple cells and exhibit desirable characteristics of spatial locality and orientation selectivity. For an orientation of $\emptyset$ and a scale $\omega$, the Gabor wavelets are defined by [19].

$$
\begin{gathered}
\psi_{\omega, \phi}(x, y)=\frac{\omega}{\sqrt{2 \pi c}} e^{-\omega^{2}\left(4 a^{2}+b^{2}\right) /\left(8 c^{2}\right)\left(e^{i a \omega}-e^{-c^{2} / 2}\right)} \\
\text { Where, } \mathrm{a}=\mathrm{x} \cos \phi+\mathrm{y} \sin \phi \quad \mathrm{b}=-\mathrm{x} \sin \phi+\mathrm{y} \sin \phi, \mathrm{c}=2.2 \\
\omega=\omega_{0} \times 2^{\mathrm{k}}, \omega_{0}=2.1, \mathrm{k}=0,1,2,3,4 \ldots
\end{gathered}
$$

To best characterize local texture properties including step and roof edge elements at an image pixel $\mathrm{I}(\mathrm{x}, \mathrm{y})$, we examine the standard "complex response" of the Gabor filter given by equation 3 for $n$ evenly spaced Gabor filter orientations.

$$
\mathrm{I}_{\text {complex }}(\mathrm{x}, \mathrm{y})=\left(\operatorname{godd}^{*} \mathrm{I}\right)(\mathrm{x}, \mathrm{y})^{2}+\left(\text { geven }^{*} \mathrm{I}\right)(\mathrm{x}, \mathrm{y})^{2}
$$

The response image for an orientation is defined as the average of the responses at the different scales. The dominant orientation is selected as the filter orientation which elicits the maximum complex response at that location.

The orientations of road texture pixels are consistence in nature. Considering the fact that road region in a given image frame consists of more than $50 \%$ of the image area, there will be very few orientations which are consistence in the nature and they can provide a very useful cue for effective voter selection. For the selection of the dominant orientations we calculate normalized histogram of orientations and consider only those orientations whose normalized score is more than or equal to a threshold value of $\mathrm{Th}_{\text {orient }} \geq 0.6$. Figure 1 shows a normalized histogram of orientations for road image. In our experiment, we discard all the pixels whose orientations are other than selected dominant orientations. The optimal value of $\mathrm{Th}_{\text {orient }}$ is selected by tuning on our test image set, where $\mathrm{Th}_{\text {orient }}=0.6$ gives maximum detection accuracy of a vanishing point. 


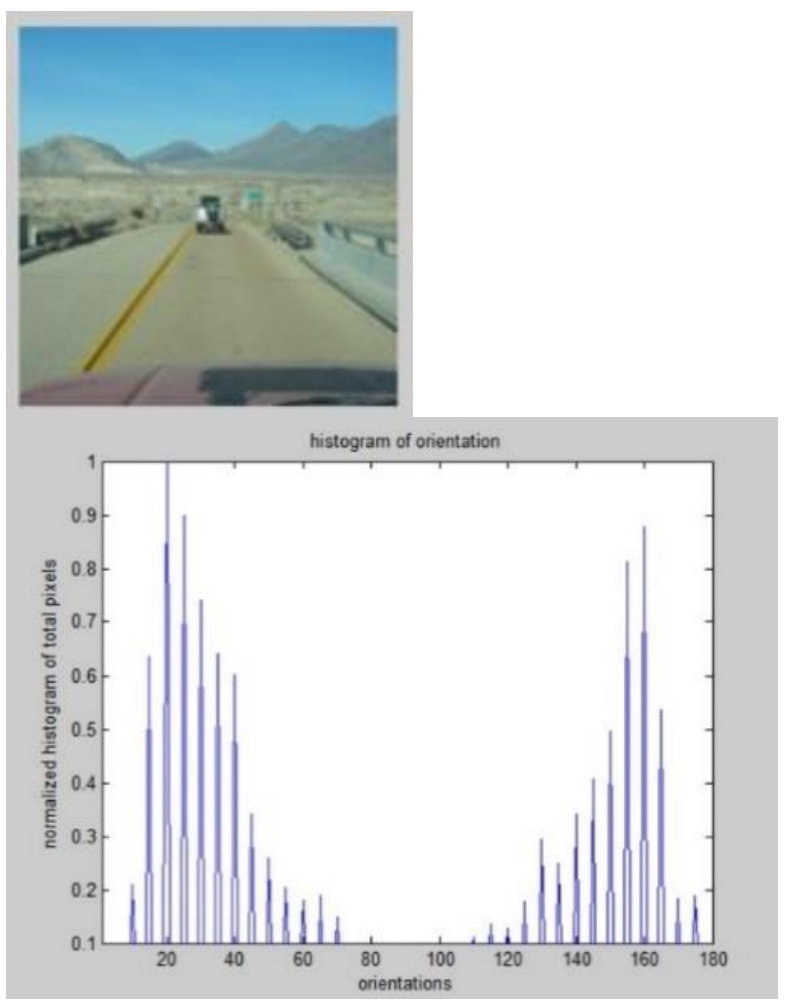

Fig. 1. Normalized Histogram of Orientation for given Road Image detected vanishing point and manually selected ground truth vanishing point is less than or equal to 10 pixels.

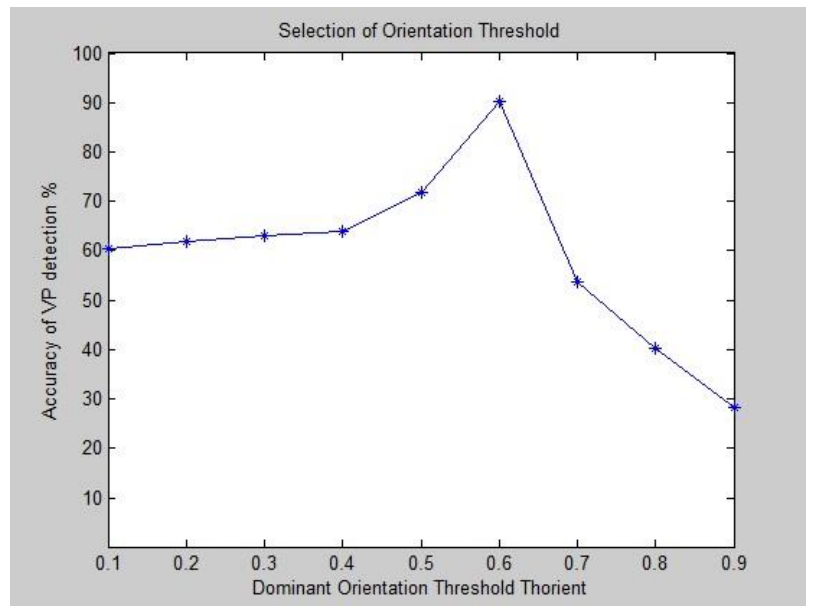

Fig. 2. Effect of $\mathrm{Th}_{\text {orient }}$ on accuracy of Vanishing Point Detection

\section{B. Selection of Candidate Pixels for Voting Process}

Accuracy and speed of a vanishing point detection process depend on no of effective voters and total no of pixels used for the voting process. Figure 3 demonstrates proposed voter selection strategy.

Figure 2 demonstrates the accuracy of the vanishing point over different $\mathrm{Th}_{\text {orient }}$ values. Here accurate vanishing point means the results for which Euclidean distance between

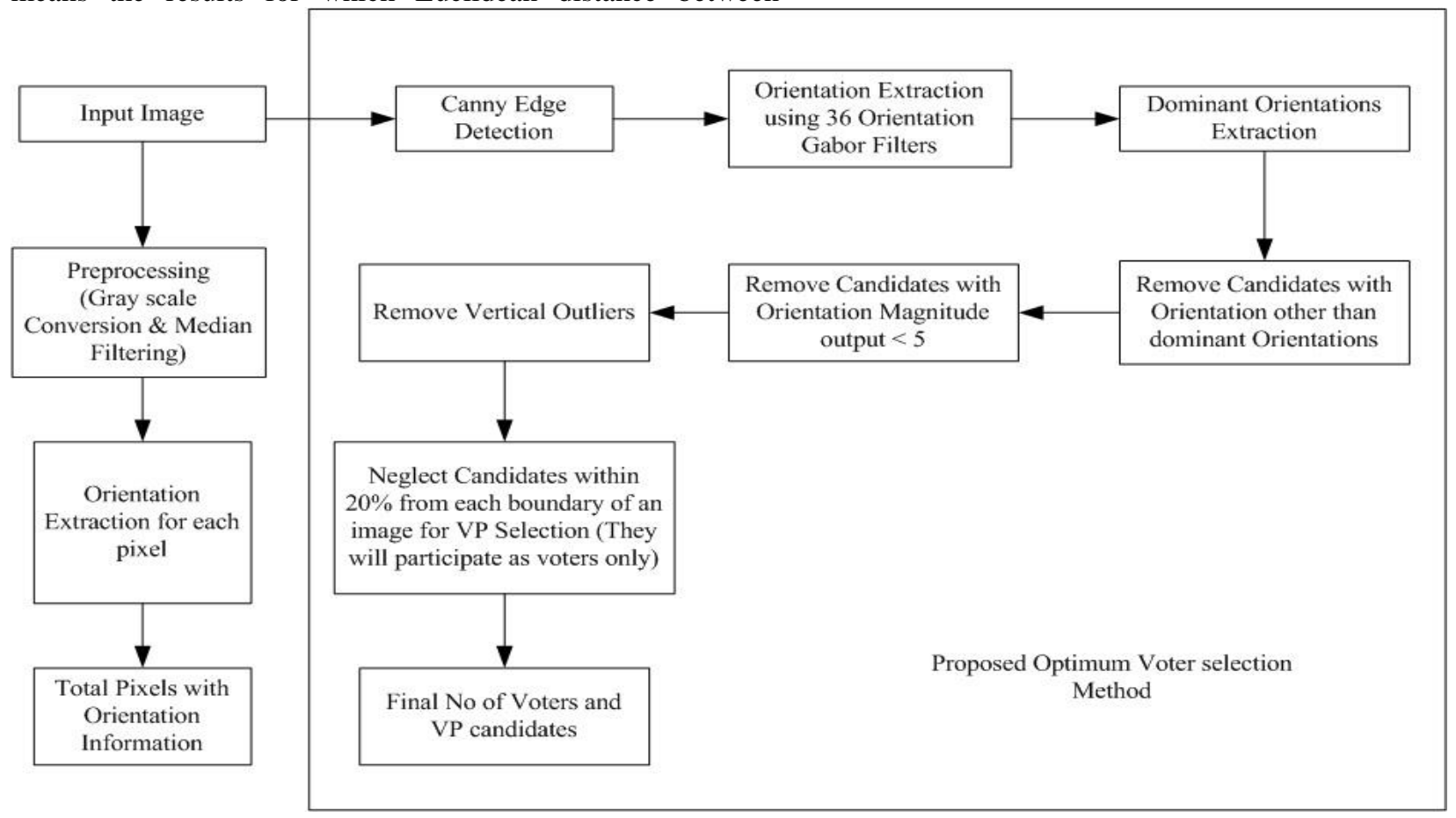

Fig. 3. Proposed Optimum Voter and Candidate Vanishing Point Selection Strategy

In proposed approach, candidate pixels are selected based on following steps:

Step I: Candidate should be an edge pixel.
Step II: Orientation of candidate pixel should be one of the dominant orientations. 
Step III: Insufficient number of voters in road images, where vanishing point is lying in bottom part of an image and major portion of an image contains sky regions, an accuracy of vanishing point decreases.

1) To address this problem we propose to identify horizon line in an image. First, the sobel horizontal filter is used to get the horizontal edge points in an image.

2) Scan all the rows from the top of an edge image and calculate a total number of horizontal edge pixels in the same row.

3) The Row which contains maximum no of edge points is selected as horizon line.

Step IV: Selection of Edge Pixels within 25\% from the Horizon Line.

1) Edge pixels above horizon line effectively do not play many roles in efficient selection of vanishing point. That's why removal of all the pixels lying within $25 \%$ portion of image from top to horizon line will not affect much on overall detection accuracy.

Step V: Removal of Outliers based on Orientation Confidence Score.

1) The confidence value is normalized magnitude response of Gabor filter with dominant orientation out of 36 orientations at that pixel.

2) Remove all outliers whose orientation confidence value is less than $2 \%$ of maximum confidence value.

Step VI: Removal of Vertical Outlier.

1) Pixels which belongs to vertical structures around the road like trees lead to the wrong erroneous detection of the vanishing point.

2) Remove pixels with orientation 90 degree in vertical length of $25 \%$ of image height are more than 20 then those pixels are considered as off road pixels due to surrounding vertical structures.

Step VII: Modification in voting area as well as Vanishing point Candidates Selection Strategy.

1) Pixels which belongs to the road surface or near road boundaries play a pivot role in true vanishing point detection. We propose to select vanishing point from selected candidates only.

2) Figure 4 shows the eligibility criteria for voter and vanishing point candidate based on location. Considering the region of interest, we neglect all the candidate pixels which belong to $20 \%$ of the portion from either side of the image.

3) As shown in figure 4, only those pixels are eligible vanishing candidates who belongs to the white portion of an image.

4) A Similar strategy is adopted for voter selection. Rather than considering semicircular region for voter selection as proposed in [17], we allow those pixels to vote which are within rectangle region of $25 \%$ of image height and $40 \%$ of image width from vanishing point candidate, as shown by a yellow portion in figure 4 which is overlapped on white image portion.

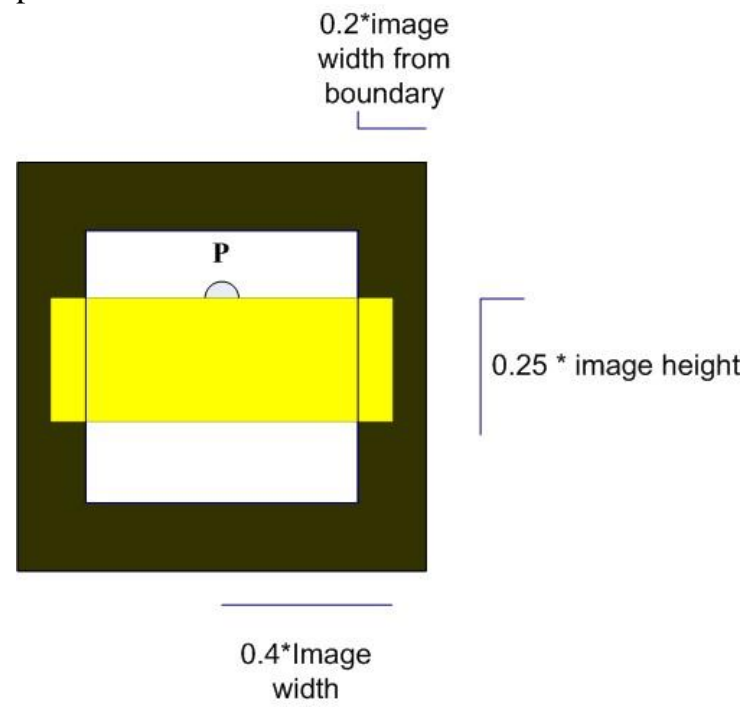

Fig. 4. Voter and Vanishing Point eligibility based on Location

As shown in figure 4 for a pixel $\mathrm{P}$ to be valid vanishing point candidate it has to be within an inner white square region, eliminating all candidates within $20 \%$ of the portion from image boundaries. Voter pixel can vote for pixel $\mathrm{P}$ if it is within the yellow rectangle. The goal of the modifying voting area is to allow maximum pixels below candidate pixel from the road surface and near road boundaries to vote for vanishing point. Remaining pixels after all above mentioned steps are considered as eligible candidates for vanishing point voting process.

Figure 5 demonstrates the selection of effective voters which will take part during the voting process. 


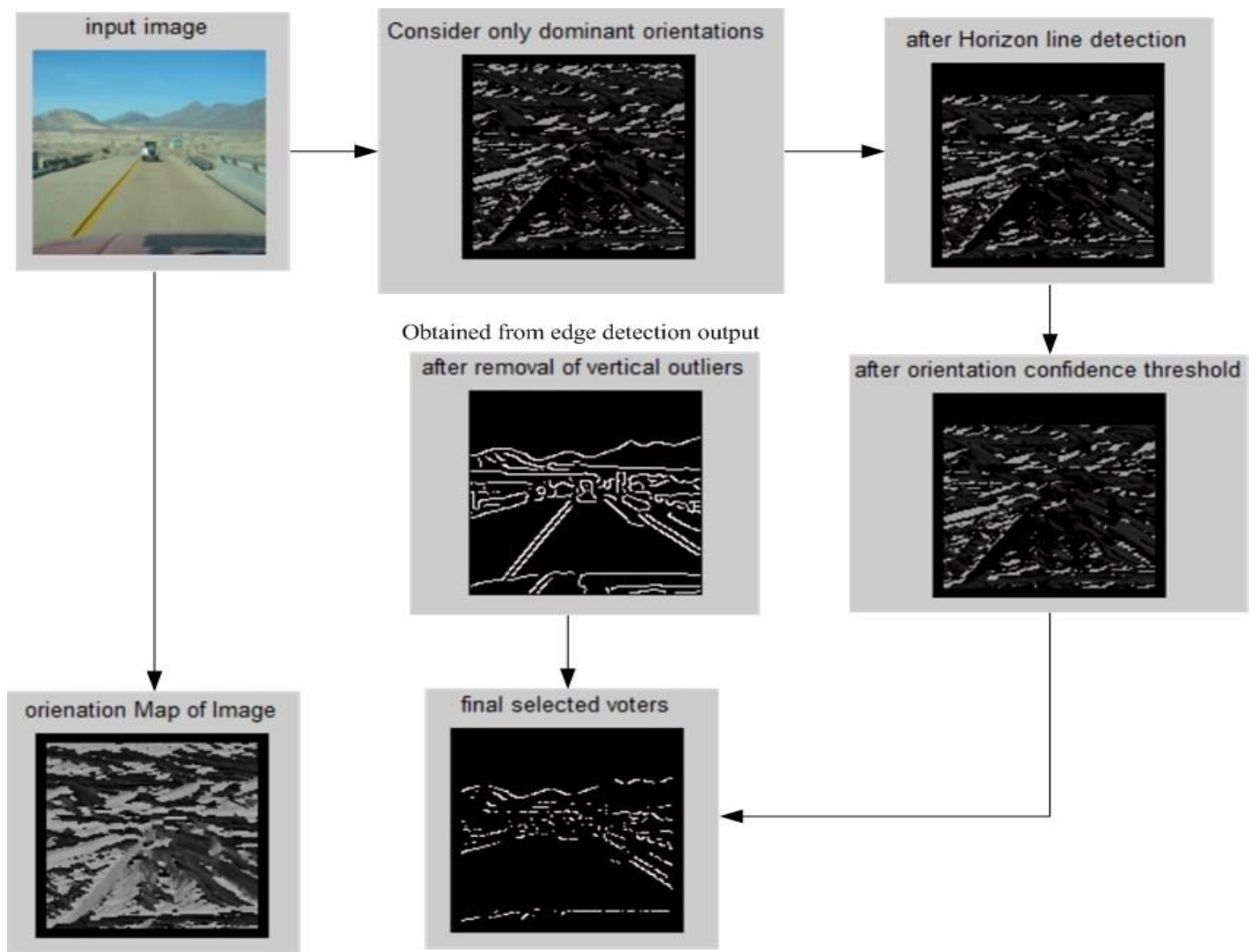

Fig. 5. Optimized Selected Voter and Candidate Pixels used for Voting Process

\section{ModifiEd Voting PROCESS}

The voting procedure is most important and critical stage of vanishing point estimation problem. Once we have computed the texture orientation at each pixel of the image, one can make these pixels vote to obtain the vanishing point. Precisely, a pixel $\mathrm{P}$ for which the texture orientation is the vector $\vec{P}$ can vote for all the pixels $\mathrm{V}$ above $\mathrm{P}$ such that the angle $\gamma=\angle((\mathrm{PV}), \overrightarrow{\mathrm{P}})<5^{\circ}$ between the direction $(\mathrm{PV})$. Here $5^{\circ}$ is the angle between two consecutive Gabor orientation filters used to extract orientations in an image. This type of voting process is referred as hard voting scheme. The only problem with this scheme is, it tends to favor points that are located in the upper portion in the image. The said problem was overcome by distance based soft voting scheme proposed by Hui Kong et al. [16-17]. Equation 4 demonstrates the voting score calculation using distance based soft voting process [16].

$$
\text { Vote }(\mathrm{P}, \mathrm{V})=\left\{\begin{array}{cc}
\frac{1}{1+[\gamma d(P, V)]^{2}} ; & \text { if } \gamma \leq \frac{1}{1+2 d(P, V)} \\
0 ; & \text { otherwise }
\end{array}\right\}
$$

In distance based soft voting process the major drawback is pixels in line with road direction but having angle $\gamma>5$ degrees are omitted from voting score calculation process which may lead to an incorrect vanishing point. In our experiments, we propose to use equation 5 for voting score calculation.

$$
\text { Vote }(\mathrm{P}, \mathrm{V})=e^{\left(-\frac{d(P, V) *(\gamma-\text { Dominantorientation })}{\text { DiagonalDistance }}\right)}
$$

Where,

DominantOrientation $=$ dominant orientation with which orientation of pixel voter orientation matches.

\section{DiagonalDistance $=$ Diagonal distance of image}

The key advantages of modified equation are:

- We allow all pixels to vote whose orientation is consistent with dominant orientations. The basic idea behind proposed modification is to remove all the voters whose orientations are not consistence with dominant orientations. This will increase the efficiency of the voting process as voters for which $\gamma>5$ degrees but their orientation is consistent with dominant orientation can take part in the voting process. All erroneous voters for which $\gamma<5$ but their orientations are not consistent with dominant orientations are neglected from voting process.

- DominantOrientation will act as a weighting parameter for voting score. If voter's orientation is one of the dominant orientation but angle $\gamma>5$ degrees, then voting score will be weighted by a value of $\gamma$. This will further enhance the effectiveness of orientation based soft voting process. 


\section{RESULT AND ANALYSIS}

Vanishing point detection algorithm is tested on standard image dataset consists of structured, semi-structured and unstructured images used by researchers in [22]. The dataset consists of images with varying intensity variations, color and texture variations and surrounding environment variations. Some image samples are shown in figure 6. All the images are resized to $128 \times 128$.
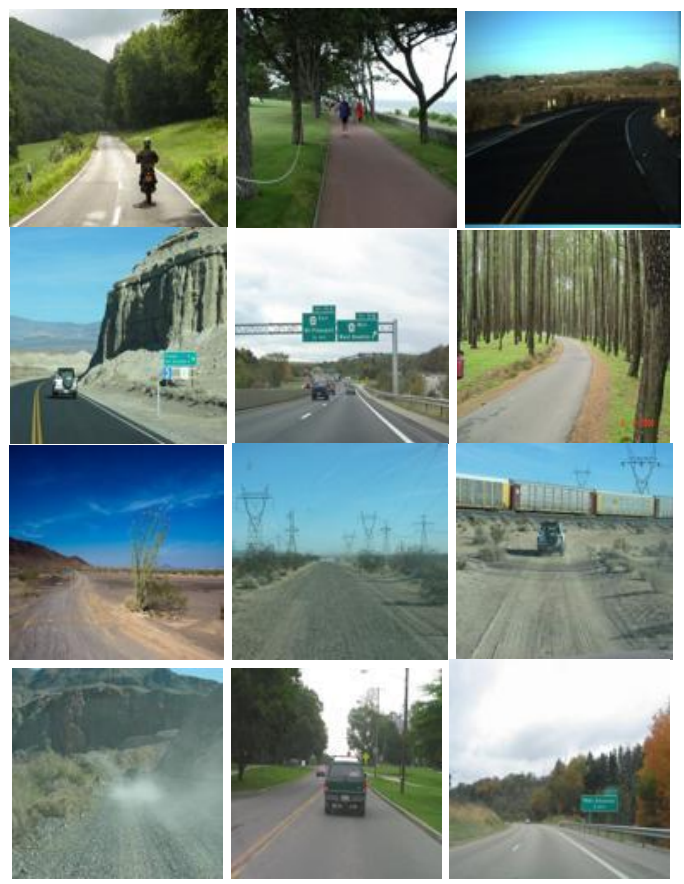

Fig. 6. Sample images from Image Database (128x 128 size)

To demonstrate the performance of proposed algorithm we generated five sets of the dataset with manually marked vanishing point by five different persons. The median filter is used to find out the final ground truth location by finding median in $\mathrm{x}$ and $\mathrm{y}$ coordinates of all marked vanishing points by different users. Figure 7 shows the results of detected vanishing point along with manually marked vanishing point as ground truth.
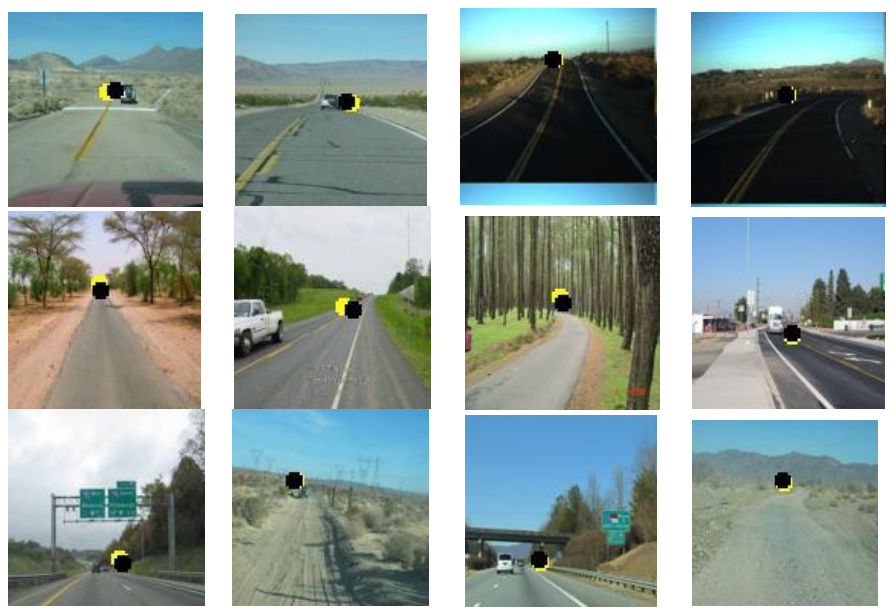

Fig. 7. Vanishing Point Detection results in yellow color with Ground Truth Positions marked with black color

Further to measure the error in vanishing point detection we have used a normalized Euclidean distance where a Euclidean distance between measured vanishing point and ground truth location is divided by the diagonal distance of the image [18].

$$
\begin{aligned}
& \text { NormError }=\frac{\|P-P 0\|}{D} \\
& \text { Where, }
\end{aligned}
$$

$\mathrm{P}=$ Detected location of vanishing point

$\mathrm{P} 0=$ Ground Truth location of vanishing point

\section{$\mathrm{D}=$ Diagonal distance of image}

Euclidean Distance of more than 10 pixels is considered as invalid vanishing point detection in our experiments. Image database consists of images with different road types and surroundings. Figure 8 shows the distance between detected vanishing point and ground truth positions against all images used. Average distance comes out to be 5.96 pixels for all test images.

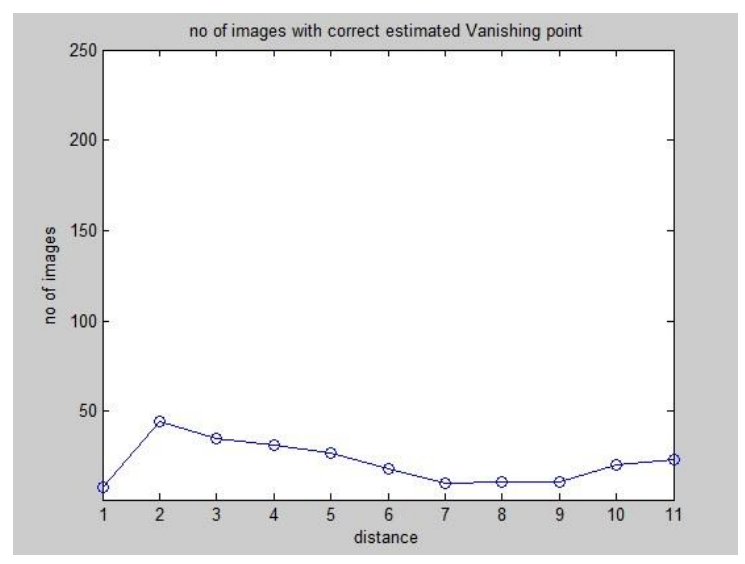

Fig. 8. Distance between detected Vanishing point and Ground Truth Position of Vanishing point for no of images

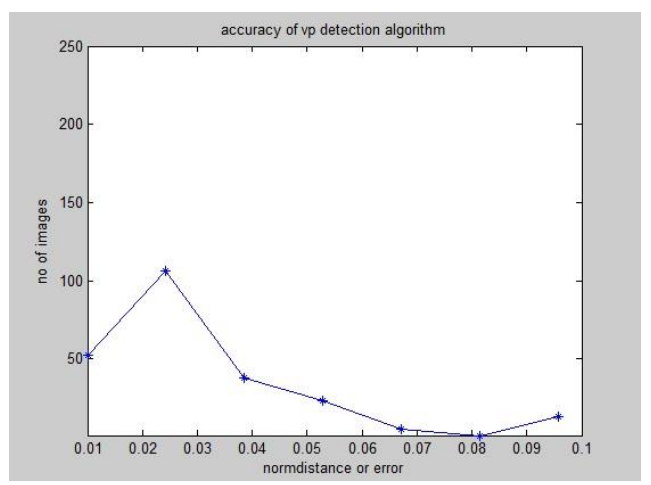

Fig. 9. Norm Distance Error for No of Images used in Experimentation

The efficiency of proposed algorithm is demonstrated in Figure 9. It shows the histogram of error in vanishing point detection against all test images. Distance error more than 0.06 is considered as the wrong detection of a vanishing point. Results are presented for 250 road images which include 
structured, semi-structured and unstructured road types. Average NormError for the proposed method is 0.033 . The accuracy of proposed system is compared with previously suggested methods. Table 1 illustrates the accuracy with modifications suggested in voter and vanishing point selection strategy as well as voting score calculation process.

TABLE I. QuANTITATIVE Evaluation OF VANIShing Point DETECTION ALGORITHM

\begin{tabular}{|c|c|c|c|}
\hline \multirow[b]{2}{*}{$\begin{array}{l}\text { VP Detection } \\
\text { Results }\end{array}$} & \multicolumn{3}{|c|}{ Quantitative Evaluation Parameters } \\
\hline & $\begin{array}{l}\text { Average } \\
\text { Euclidean } \\
\text { Distance }\end{array}$ & $\begin{array}{l}\text { Average } \\
\text { Accuracy } \\
\%\end{array}$ & $\begin{array}{l}\text { Average } \\
\text { Norm } \\
\text { Error }\end{array}$ \\
\hline Proposed Method & 5.964 & 90.33 & 0.033 \\
\hline $\begin{array}{l}\text { Without horizon line } \\
\text { Detection }\end{array}$ & 7.2351 & 86.97 & 0.04 \\
\hline $\begin{array}{l}\text { Without } \\
\text { modification in } \\
\text { Voting score } \\
\text { calculation (use of } \\
\text { only distance based } \\
\text { soft voting) }\end{array}$ & 7.34 & 82.77 & 0.0405 \\
\hline
\end{tabular}

Quantitative comparison of proposed vanishing point detection algorithm with previously proposed methods for vanishing point detection based on Gabor filter orientation extraction is shown in table 2 .

TABLE II. ACCURACY COMPARISON WITH PREVIOUSLY PROPOSED METHODS FOR VANISHING POINT DETECTION

\begin{tabular}{|l|l|l|l|l|l|}
\hline & $\begin{array}{l}\text { Edge- } \\
\text { Based } \\
\text { Method } \\
{[12]}\end{array}$ & $\begin{array}{l}\text { Rasmussen } \\
{[15]}\end{array}$ & $\begin{array}{l}\text { Kong } \\
\text { et. al } \\
{[17]}\end{array}$ & $\begin{array}{l}\text { Peyman } \\
\text { et. al[18] }\end{array}$ & $\begin{array}{l}\text { Proposed } \\
\text { withod } \\
\text { optimized } \\
\text { voter } \\
\text { selection } \\
\text { strategy }\end{array}$ \\
\hline $\begin{array}{l}\text { Norm } \\
\text { Error }\end{array}$ & 0.171 & 0.0903 & 0.052 & 0.036 & $\mathbf{0 . 0 3 3}$ \\
\hline
\end{tabular}

\section{CONCLUSION}

Less computational cost and speed are the two major concerns in existing vanishing point detection algorithms [14$18,20,21]$. Speed and computational cost of vanishing point detection algorithm mainly depend on total no of pixels used during voting process. If we can optimize the no of pixels and select only most efficient pixels during the voting process the performance can be improved. With this motivation, we presented an optimized strategy to select most effective candidates for the vanishing point detection process. Selection strategy of efficient voter depends on the efficient selection of dominant orientation and removal of outlier pixels which may lead to inaccurate results. Once efficient pixels are selected, second modification suggested here is location based eligible candidate selection for vanishing point and voter. Finally, the use of combined soft voting and dominant orientation based voting score calculation increases the accuracy of vanishing point detection. Error in vanishing point occurs when vanishing points are located in the bottom part of an image, and large portion of an image contains sky region. To overcome this problem we suggest a very simple and fast horizon line detection based outlier removal method to reject the outliers which may lead to inaccurate results. Furthermore, a series of qualitative and quantitative results are presented to validate the accuracy of proposed algorithm. The proposed algorithm outperforms previously suggested methods regarding accuracy using only $2.36 \%$ of total image pixels i.e. average 360 pixels from 128x128 images.

\section{REFERENCES}

[1] Y. He, H. Wang and B. Zhang, "Color-based road detection in urban traffic scene.” IEEE Trans. Intell. Transp. Syst., Vol. 5(4), pp.309-318, 2004.

[2] G. Finlayson, S. Hordley, C. Lu and M. Drew, "On the removal of shadows from images.” IEEE Trans. Pattern Anal. Mach. Intell., Vol. 28, pp. 59-68, 2006.

[3] C. Tan, T. Hong, T. Chang and M. Shneier, "Color model-based realtime learning for road following.” Proc. IEEE Intell. Transp. Syst. The conference, pp. 939-944, 2006.

[4] Tsung-Ying Sun, Shang-Jeng Tsai and Chan V, "HSI color model based lane-marking detection." Proc. Intelligent Transportation Systems Conference, pp. 1168-1172, September 2006.

[5] D. Puig and M. A. Garcia, "Automatic Texture Feature Selection for Image Pixel Classification. Pattern Recognition.” Vol. 39, pp.1996 2009, 2006

[6] D. Puig, M. A. Garcia and J. Melende, "Application- Independent Feature Selection for Texture Classification." Pattern Recognition,Vol. 43, pp. 3282-3297, 2010

[7] Alvarez J. and Ĺopez A. M. Road Detection Based on Illuminant Invariance. IEEE Trans. Intelligent Transportation Systems, Vol. 12, pp. 184-193, 2011.

[8] K. H. Chen and W. H. Tsai, "Vision-Based Autonomous Land Vehicle Guidance in Outdoor Road Environments Using Combined Line and Road Following Techniques.” Journal of Robotic Systems, Vol. 14(10), pp. 711-728, 1997.

[9] B. Yu and A. K. Jain, "Lane boundary detection using a multiresolution Hough transform." Proc. IEEE Int. Conf. Image Processing, vol. 2, pp. 748-751, 1997.

[10] Dezhi Gao, Wei Li, Jianmin Duan and Banggui Zheng, "A practical method of road detection for intelligent vehicle." Proc. IEEE International Conference on Automation and Logistics, pp. 980,985, 5-7 Aug. 2009.

[11] Tuytelaars T., Van Gool L., Proesmans M. and Moons, T, "The cascaded Hough transform as an aid in aerial image interpretation." Proc. International Conference on Computer Vision, Vol. 4, pp. 67-72, Jan 1998.

[12] Y.Wang, E. K. Teoh and D. Shen, "Lane detection and tracking using BSnake." Image Vis. Comput., Vol 22(4), pp. 269-280, 2004.

[13] W. T. Freeman and E. H. Adelson, " The design and use of steerable filters.” IEEE Trans Pattern Anal. Mach. Intell. Vol. 13(9), pp. 891-906, 1991.

[14] C. Rasmussen, "Grouping dominant orientations for ill-structured road following." Proc. IEEE Conf. Comput. Vis. Pattern Recog., pp. 470-477, 2004.

[15] C. Rasmussen, "Texture-based vanishing point voting for road shape estimation.” Proc. British Machine Vision Conf., 2004.

[16] H. Kong, J.Y. Audibert and J. Ponce. Vanishing point detection for road detection. Proc. IEEE Conf. Computer Vision Pattern Recognition, 2009, 96-103.

[17] H. Kong, J.Y. Audibert and J. Ponce, "General road detection from a single image." IEEE Trans. On Image Processing, Vol. 19(8), pp. 22112220, 2010.

[18] Moghadam, P. Starzyk J.A. and Wijesoma W.S., "Fast Vanishing-Point Detection in Unstructured Environments." IEEE Trans. On Image Processing, Vol. 21(1), pp. 425-430, 2012.

[19] T. Lee, "Image representation using 2d Gabor wavelets." IEEE Trans. Pattern Anal. Mach. Intell., Vol. 18(10), pp. 959-971, 1996.

[20] Xiqun Lu, "A new efficient vanishing point detection from single image." Proc. ICASSP, pp. 901-904, 2012.

[21] Cheng Xu, Youqi Cai, Zheng Tian, Tao Li and Yan Liu, "Principal Orientation and generalized vanishing point based road detection." Proc. 
(IJACSA) International Journal of Advanced Computer Science and Applications, Vol. 7, No. 8, 2016

International Conference on Computer Science and Service System, pp. 394-397, 2014.
[22] http://web.mit.edu/huikong/www/Road_Detection_files/roadImgs_final.r ar 